\title{
Histopathological characteristic of chronic hbv infection in Bulgaria
}

\author{
Radina Ivanova, Asen Aleksiev, Dimitar Popov, \\ Donika Krasteva, Deian Jelev, Krasimir Antonov, \\ Lyudmila Mateva, Zahariy Krastev
}

Clinic of Gastroenterology, Department of Internal Medicine, Faculty of Medicine, Medical University Sofia, UMHAT "St. IvanRilski ", Sofia, Bulgaria;

\begin{abstract}
Introduction

The course of HBV infection is influenced by many factors. Various guidelines have recommended liver biopsy in different groups of CHB patients.
\end{abstract}

\section{Aim}

The aim of our study was to evaluate the histomorphological findings in Bulgarian patients with chronic HBV infection.

\section{Materials and methods}

A total of 335 patients with chronic HBV infection were evaluated. Two hundred and thirty one patients had liver biopsies and the remaining 104 patients were with liver cirrhosis. The grades of activity and stages of fibrosis were scored according to METAVIR

\section{Results}

The proportion of the patients with mild activity grade (40.7\%) was the highest. Regarding the fibrosis stages, the proportions of cases with mild and moderate stages of fibrosis were similar (32.5\% and $31.6 \%$, respectively) and higher compared to those of cases with advanced fibrosis stages (22.1\% and 
13.9 for F3 and F4 stages, respectively). There was a trend for an increase of the mean age of patients from the subgroups with mild to severe activity grades. The mean age of the patients with LC was significantly higher compared to that of the subgroups of patients with different activity grades, respectively $(p=0.001)$. There were significant differences between the mean ages of patients with $F 1$, $F 2$ or F3 stages and those of patients with F4 and LC $(p=0.015-0.0001)$. No sex difference was found in the comparison of the mean ages of the patients with the individual grades of activity or stages of fibrosis.

\section{Conclusion}

About 30 percent of Bulgarian patients with $C H B$ are cirrhotic before starting antiviral therapy and 10 years older compared to the non-LC CHB patients. Almost three quarters of all patients with CHB were males. Severe histomorphological activity was found in only $1 / 5$ of the patients with CHB and advanced fibrosis in 1/3 of them. There was a trend for an increase of the mean age of patients with CHB from the subgroups with mild to severe activity grade and from F1 to F4 stage, respectively.

\section{Keywords: hepatitis B, fibrosis, inflammation, age, histopathology, cirrhosis}

\section{Introduction}

About 350 millions live with chronic hepatitis B infection (CHB). The course of HBV infection is a dynamic process and is influenced by many factors including viral, host, and exogenous factors. Various guidelines have recommended liver biopsy in different groups of $\mathrm{CHB}$ patients. The aim of our study was to evaluate the histomorphological findings in Bulgarian patients with chronic HBV infection

\section{Materials and methods}

A total of 335 patients with chronic HBV infection, diagnosed and treated at Clinic of Gastroenterology, University Hospital St. Ivan Rilski (Sofia, Bulgaria) during the last 20 years were included in the study. The diagnosis of chronic hepatitis B was based on standard clinical, laboratory, and other investigations,including abdominal ultrasonography with Doppler ultrasound. Two hundred and thirty one patients had liver biopsies but in the remaining 104 patients there were clinical data for liver cirrhosis (LC) and no indications for liver biopsy.

All liver biopsy samples were processed routinely and sections, stained with hematoxylin eosin and Masson's trichrome, were morphologically assessed. The grades of activity and stages of fibrosis were scored according to METAVIR (1) as follows: A0 - no activity, A1 - mild, A2 - moderate or A3-severe activity, and F1 - mild fibrosis (portal fibrosis without septa), F2 - moderate fibrosis (portal fibrosis with few septa), F3 - severe fibrosis (numerous septa without cirrhosis) or F4 cirrhosis.

Statistical analysis of the data was performed using SPSS. The descriptive, Student's t-test, nonparametric and parametric analyses were done. In all analyses $p$ values lower than 0.05 were considered to be statistically significant. 


\section{Results}

\section{Characteristic of the patients with chronic HBV infection}

A total of 335 patients with CHB were included in the study (table I). Of these, there were 231 patients, who had liver biopsies, in the non-LC CHB group with a mean age of $41.63 \pm 11.41$ years and 104patients in the LC group, with a mean age of $50.90 \pm 11.74$ years. There were $177(76.6 \%)$ and 77 males $(69.2 \%)$ in the non-LC and LC groups, respectively. We found a significant difference between the ages of the patients in the two groups $(p<0.0001)$, but without sex difference.

Histomorphological assessment of liver biopsy samples of the patients with $\mathrm{CHB}$ showed that the proportion of the patients with mild activity grade (40.7\%) was the highest, following by those of moderate (30.7\%) and severe activity grade (20.8\%), and the proportion of cases with no inflammation was the lowest $(7.8 \%)$, (table 2). Regarding the fibrosis stages, the proportions of cases with mild and moderate stages of fibrosis were similar (32.5\% and 31.6\%, respectively) and higher compared to those of cases with advanced fibrosis stages (22.1\% and 13.9 for F3 and F4 stages, respectively).

Table. 1. Characteristic of Bulgarian patients with chronic HBV infection

\begin{tabular}{|l|l|l|l|}
\hline Parameter & $\begin{array}{l}\text { Non-LC CHB } \\
(\mathrm{n}=231)\end{array}$ & $\begin{array}{l}\text { LC } \\
(\mathrm{n}=104)\end{array}$ & $\mathrm{p}$ \\
\hline Age, years (Mean \pm SD) & $41.63 \pm 11.41$ & $50.90 \pm 11.74$ & 0.0001 \\
\hline Sex (male/female) & $177 / 54$ & $72 / 32$ & NS \\
\hline
\end{tabular}

Table 2. Liver histomorphology in patients with chronic HBV infection

\begin{tabular}{|c|c|c|}
\hline Histomorphology & $\mathrm{N}$ & Percent \\
\hline \multicolumn{3}{|c|}{ Activity grades (METAVIR), n=231 } \\
\hline $\mathrm{AO}$ & 18 & 7.8 \\
\hline A1 & 94 & 40.7 \\
\hline A2 & 71 & 30.7 \\
\hline A3 & 48 & 20.8 \\
\hline \multicolumn{3}{|c|}{ Fibrosis stages (METAVIR), $\mathrm{n}=231$} \\
\hline F1 & 75 & 32.5 \\
\hline F2 & 73 & 31.6 \\
\hline F3 & 51 & 22.1 \\
\hline F4 & 32 & 13.9 \\
\hline
\end{tabular}


Relationships between the histopathological findings and patients characteristic with chronic HBV infection

The mean age of subgroups of patients according to the activity grades and fibrosis stages and LC was given on fig. 1 and 2. There was a trend for an increase of the mean age of patients from the subgroups with mild to severe activity grades but without significant differences in their comparison (fig. 1). The mean age of the patients with LC was significantly higher compared to that of the subgroups of patients with A0, $A 1, A 2$ or A3 activity grades, respectively $(p=0.001)$. The mean age values of the patients from the subgroups with F1 to F4 stage showed a similar tendency (fig. 2). There were significant differences between the mean ages of patients with F1, F2 or F3 stages and those of patients with F4 and LC, respectively $(p=0.015-0.0001)$.

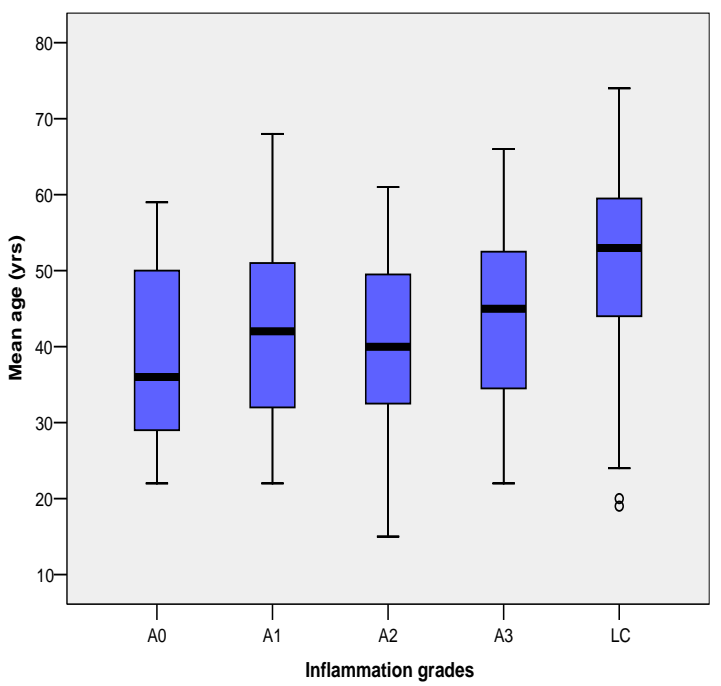

Figure 1. Mean age of the patients with CHB according to the grade of activity and LC.

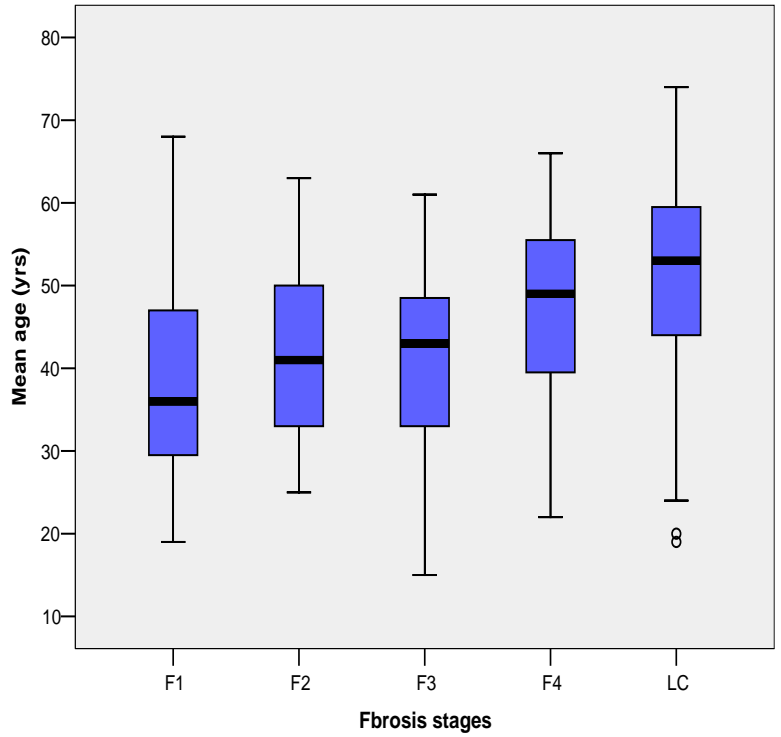

Figure 2. Mean age of the patients with CHB according to the fibrosis stage and LC. 
The proportions of male and female patients according to the activity grades and fibrosis stages were given on table 3. There were no significant differences in the proportions of males and females from the subgroups with A0 to A3 activity grades and F1 to F4 stages, respectively. No sex difference was found also in the comparison of the mean ages of the patients with the individual grades of activity or stages of fibrosis, respectively.

Table 3. Sex distribution of the patients with chronic HBV infection according to the grades of activity and stages of fibrosis.

\begin{tabular}{|l|l|l|}
\hline Histomorphology of CHB & $\begin{array}{l}\text { Males } \\
\text { N (\%) }\end{array}$ & $\begin{array}{l}\text { Females } \\
n(\%)\end{array}$ \\
\hline Activity grades & $13(72.2)$ & $5(27.8)$ \\
\hline A0 $(n=18)$ & $73(77.7)$ & $21(22.3)$ \\
\hline A1 $(n=94)$ & $52(73.2)$ & $10(26.8)$ \\
\hline A2 $(n=71)$ & $39(81.3)$ & $9(18.8)$ \\
\hline A3 $(n=48)$ & \multicolumn{2}{|l|}{} \\
\hline Fibrosis stages & $20(26.7)$ \\
\hline F1 $(n=75)$ & $55(73.3)$ & $20(27.4)$ \\
\hline F2 $(n=73)$ & $53(72.6)$ & $6(11.8)$ \\
\hline F3 $(n=51)$ & $45(88.2)$ & $8(25.0)$ \\
\hline F4 $(n=32)$ & $24(75.0)$ &
\end{tabular}

\section{Discussion}

In Bulgaria there were no summarized data for the histomorphological characteristic of chronic hepatitis B. The demographic characteristic of our series of patients with CHB suitable for antiviral treatment represents an extract of this disease in our country during the last two decades (2).

Although liver biopsy gives up its place on other surrogate markers in the treatment algorithms in patients with $\mathrm{CHB}$, it reflects the most exactly the pathological process of the disease, including its transition from mild changes to cirrhosis (3). In this study we characterized the histomorphological findings on liver biopsies from patients with CHB during the last 20 years using METAVIR activity and fibrosis scores for chronic hepatitis, which are widely accepted methods for standardization of morphological changes. It has to mention that the portion of patients with clinical data for cirrhosis at the time of diagnosis of chronic hepatitis $B$ infection was nearly $30 \%$. This result showed once again the silent progression of the disease in some cases. In our patients with CHB we found that mild activity was more common compared to the mild fibrosis ( $40 \%$ and $30 \%$, respectively). On the opposite, severe activity grade was established only in $20 \%$ of the cases with $\mathrm{CHB}$ and advanced fibrosis in $36 \%$. Our results were similar to those of one Chinese study, including almost ten times higher number of patients with $\mathrm{CHB}(n=2300)(4)$. The authors reported that severe activity was present in $23-28 \%$ among the cases with elevated liver enzymes but only in $10 \%$ of the cases with normal liver enzymes. Advanced fibrosis was found in $21-28 \%$ in patients with elevated enzymes (4). Another one Asiatic study of 950 treatment naïve CHB patients, using transient elastography, estimated severe fibrosis in $34 \%$, which result was almost overlapping with ours (5). 
In our study we found no sex difference in the severity of activity and fibrosis although the most of patients were males. Regarding the age of the patients with $\mathrm{CHB}$, there was a tendency for increasing the severity of activity and fibrosis with advance of the age. In the maintenance of this tendency is also the higher mean age of the LC group of patients compared to the one of non-LC CHB patients. Similarly, severe fibrosis was seen with increasing age from $20 \%$ in patients $<25$ years to $81 \%$ in those $>65$ years (5). We can summarized that there were no substantial differences between the histomorphological findings of our and Asiatic patients with $\mathrm{CHB}$. Our results confirmed once again the important role of liver biopsy in the evaluation of the activity grades and fibrosis stages in patients with chronic hepatitis $B$, giving valuable information in the choice of treatment (3).

\section{Conclusion}

About 30 percent of Bulgarian patients with $\mathrm{CHB}$ are cirrhotic before starting antiviral therapy. The cirrhotics were roughly 10 years older compared to the non-LC CHB patients, without sex difference. Almost three quarters of all patients with CHB were males. Severe histomorphological activity was found in only $1 / 5$ of the patients with $\mathrm{CHB}$ and advanced fibrosis in $1 / 3$ of them. There was a trend for an increase of the mean age of patients with CHB from the subgroups with mild to severe activity grade and from F1 to F4 stage, respectively.

\section{References}

1. Bedossa P, Poynard T, The French METAVIR Cooperative Study Group. An algorithm for grading activity in chronic hepatitis C. Hepatology 1996;24: 289-29.

2. Krasteva D, Boyanova Y, Mateva L, Krastev Z. Demographics of the chronic HBV infection suitable for antiviral treatment-20 years of experience in a single Bulgarian centre. Medinform 2018, 2, 853857.

3. Mani H, Kleiner DE. Liver biopsy findings in chronic hepatitis B. Hepatology. 2009 May;49(5 Suppl):S61-71.

4. Tan Y, Ye Y, Zhou X, Chen L, Wen D. Age as a predictor of significant fibrosis features in HBeAgnegative chronic hepatitis $B$ virus infection with persistently normal alanine aminotransferase. PLoS One. 2015;10(4):e0123452.

5. Fung J, Lai CL, But D, Wong D, Cheung TK, Yuen MF. Prevalence of fibrosis and cirrhosis in chronic hepatitis B: implications for treatment and management. Am J Gastroenterol. 2008;103(6):1421-6.

\section{Corresponding author:}

Radina Ivanova,

Clinic of Gastroenterology,

Department of Internal Medicine,

Faculty of Medicine

Medical University - Sofia

UMHAT "St. IvanRilski ",

Sofia, Bulgaria

email: radinaivanova@abv.bg 